\title{
Relationship between pulmonary arterial resistance and compliance among patients with pulmonary arterial hypertension and congenital heart disease
}

\author{
Jun Muneuchi, MD, ${ }^{\mathrm{a}}$ Yusaku Nagatomo, MD, ${ }^{\mathrm{a}}$ Mamie Watanabe, MD, ${ }^{\mathrm{a}}$ Kunihiko Joo, MD,
}

Tatsushi Onzuka, MD, ${ }^{\mathrm{b}}$ Yoshie Ochiai, MD, ${ }^{\mathrm{b}}$ and Kunitaka Joo, MD ${ }^{\mathrm{a}}$

\begin{abstract}
Background: It is unknown whether changes in pulmonary arterial resistance (Rp), pulmonary arterial compliance $(\mathrm{Cp})$, and the product of $\mathrm{Rp}$ and $\mathrm{Cp}$ (the $\mathrm{RC}$ time) in patients with pulmonary arterial hypertension (PAH) are related to an increase in pulmonary blood flow. The aim of this study is to clarify relationships between these parameters before and after corrective surgery among patients with PAH and congenital heart disease.
\end{abstract}

Methods: We performed cardiac catheter examinations and compared $\mathrm{Rp}, \mathrm{Cp}$, and the RC time before and after corrective surgery in 100 infants (53 boys) with PAH related to ventricular septal defect.

Results: Median age at surgery was 2.9 (0.6-28.5) months. Preoperative values of the ratio of pulmonary to systemic blood flow (Qp/Qs), systolic pulmonary arterial pressure (PAP), Rp, Cp, and the RC time were 3.5 (2.7-4.3), 65 (56-70) $\mathrm{mm} \mathrm{Hg}$, 2.18 (1.64-3.19) Wood unit $/ \mathrm{m}^{2}, 2.67$ (2.01-3.38) $\mathrm{mL} / \mathrm{mm} \mathrm{Hg} / \mathrm{m}^{2}$, and $0.36(0.31-$ 0.40 ) seconds, respectively. Postoperative systolic PAP, $\mathrm{Cp}$, and the RC time were significantly decreased to 26 (23-29) $\mathrm{mm} \mathrm{Hg}, 1.96(1.77-2.26) \mathrm{mL} / \mathrm{mm} \mathrm{Hg} / \mathrm{m}^{2}$, and $0.31(0.26-0.36)$ seconds, respectively, although $\mathrm{Rp}$ remained unchanged at 2.53 (2.06-3.31) Wood unit $/ \mathrm{m}^{2}$. The relationship between $\mathrm{Rp}$ and $\mathrm{Cp}$ was inversely related before and after surgery, and the Rp-Cp coupling curve was shifted downward after surgery. In addition, lower preoperative $\mathrm{Cp}$ (partial regression coefficient $=-3.35 ; P=.001$ ) and preterm delivery (partial regression coefficient $=6.28 ; P=.02$ ) were independently related to postoperative higher systolic PAP.

Conclusions: Rp-Cp coupling depends on both the amount of pulmonary blood flow and the condition of the pulmonary vasculature. Lower preoperative $\mathrm{Cp}$ is an independent predictive factor to predict higher postoperative pulmonary systolic pressure leading to an increase in right ventricular workload. It is necessary to assess Rp-Cp coupling before surgery among patients with PAH related to congenital heart disease. (J Thorac Cardiovasc Surg 2016;152:507-13)

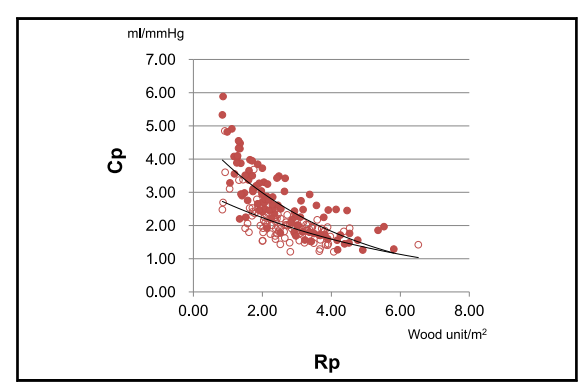

The changes in pulmonary arterial resistance and compliance before and after surgery.

\section{Central Message}

Pulmonary arterial compliance and RC-time constant were reduced after surgery for pulmonary hypertension as a result of high pulmonary flow.

\section{Perspective}

Pulmonary arterial resistance and compliance are inversely related and fixed during therapy for PAH. However, there is little information about these parameters in PAH related to congenital heart disease, which is the most common cause of PAH in children. We clearly show the relationship between $\mathrm{Rp}$ and $\mathrm{Cp}$ and suggest that preoperative $\mathrm{Cp}$ can be a predictor of postoperative pulmonary arterial pressure.

See Editorial Commentary page 514.
In the pulmonary circuit, it is important to consider the following 2 parameters: pulmonary arterial resistance (Rp), which refers to the resistance encountered by blood

\footnotetext{
From the Departments of ${ }^{\mathrm{a} P e d i a t r i c s}$ and ${ }^{\mathrm{b}}$ Cardiovascular Surgery, Japan Community Healthcare Organization, Kyushu Hospital, Kitakyushu, Fukuoka, Japan. Received for publication May 8, 2015; revisions received March 16, 2016; accepted for publication March 27, 2016; available ahead of print May 14, 2016. Address for reprints: Jun Muneuchi, MD, Department of Pediatrics, Japan Community Healthcare Organization, Kyushu Hospital, 1-8-1, Kishinora, Yahatanishiku, Kitakyushu, Fukuoka, 806-8501, Japan (E-mail: jmune@msn.com). $0022-5223 / \$ 36.00$

Copyright (c) 2016 by The American Association for Thoracic Surgery http://dx.doi.org/10.1016/j.jtcvs.2016.03.080
}

as it flows through the pulmonary vasculature; and pulmonary arterial compliance $(\mathrm{Cp})$, which refers to the elasticity and extensibility of vessels, representing the Windkessel model proposed by Otto Frank in 1899. ${ }^{1}$ There is an inverse relationship between $\mathrm{Rp}$ and $\mathrm{Cp}$, and the $\mathrm{Rp}-\mathrm{Cp}$ coupling reflects the condition of the pulmonary vasculature in

Scanning this QR code will take you to the article title page.

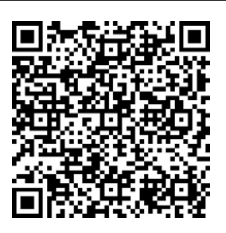




\section{Abbreviations and Acronyms \\ $\mathrm{Cp} \quad=$ pulmonary arterial compliance \\ $\mathrm{CTEPH}=$ chronic thromboembolic pulmonary hypertension \\ IPAH = idiopathic pulmonary arterial hypertension \\ PAH = pulmonary arterial hypertension \\ PAP = pulmonary arterial pressure \\ $\mathrm{Qp} \quad=$ pulmonary blood flow \\ Qs = systemic blood flow \\ $\mathrm{RC}=$ resistor-capacitor \\ $\mathrm{Rp} \quad=$ pulmonary arterial resistance}

patients with pulmonary arterial hypertension $(\mathrm{PAH}) .^{2-5}$ The product of $\mathrm{Rp}$ and $\mathrm{Cp}$, called the resistor-capacitor (RC) time, represents the exponential pressure decay in the pulmonary artery during diastole, which is potentially useful in the study of pulmonary circulation as an independent factor of right ventricular afterload and explains the changes of cardiac index better than other parameters. ${ }^{4}$ The RC time has previously been shown to be fixed during therapy for PAH; however, it is unknown whether this is true in patients with PAH related to left-to-right shunt, in which pulmonary blood flow changes significantly before and after the surgical procedure. Therefore, we investigated the changes in the RC time and Rp-Cp coupling before and after corrective surgery in infants with PAH related to a ventricular septal defect, because that is the stereotypical congenital heart disease.

\section{PATIENTS AND METHODS \\ Participants}

We included 100 children with PAH associated with a ventricular septal defect who underwent corrective surgery between 2000 and 2014. All the patients had symptoms related to cardiac failure, and an accentuated pulmonic second heart sound was noted in addition to findings of right ventricular hypertrophy on electrocardiogram and echocardiogram, suggesting $\mathrm{PAH}$. In our standard institutional clinical practice, cardiac catheterizations are indicated before and after corrective surgery to confirm the improvement in PAH in infants with left-to-right congenital heart disease. We retrospectively obtained right heart catheter measurements from clinical records before and after corrective surgery. We excluded patients with other cardiac lesions, such as patent ductus arteriosus, moderate or severe valve regurgitation, right or left ventricular outflow obstruction, arch anomaly, and left ventricular dysfunction (ejection fraction $<0.6$ ), because we intended to determine the relationship between $\mathrm{Rp}$ and $\mathrm{Cp}$ in PAH relevant to the increase in pulmonary blood flow (Qp). We also excluded patients with significant residual left-to-right shunt after corrective surgery. The institutional ethics committee approved the study, and all patients or their guardians gave informed consent.

\section{Cardiac Catheterization and Data Collection}

Cardiac catheter examinations were performed before and after corrective surgery to evaluate pulmonary hemodynamics. All catheterization measurements were performed in the supine position under sedation with thiamylal sodium using fluoroscopic guidance with standard techniques. Pressures were measured using a Berman angiographic catheter or a Swan-Ganz catheter (Gadelius Medical K.K., Tokyo, Japan) at end expiration. If possible, left atrial pressure was measured through the foramen ovale, and if not possible, pulmonary capillary wedge pressure was measured to calculate transpulmonary pressure. PAH is confirmed if the mean pulmonary arterial pressure (PAP) is $>25 \mathrm{~mm} \mathrm{Hg}$ or the ratio of mean PAP and systemic arterial pressure is $>0.3$. Qp was calculated using the Fick principle. Rp was calculated as the difference between mean PAP and left atrial pressure, or pulmonary arterial capillary wedge pressure was divided by Qp. Pulmonary stroke volume was calculated as Qp divided by the heart rate during examination. $\mathrm{Cp}$ was calculated as the pulmonary stroke volume divided by the pulmonary arterial pulse pressure (the difference between systolic and diastolic PAPs). The RC time was the product of $\mathrm{Rp}$ and $\mathrm{Cp}$ as described previously.

\section{Statistical Analysis}

Statistical analysis was performed using the analysis toolpack in Microsoft Office Excel add-in software. Values are expressed as the median following the first and third quartiles, and variables before and after surgery were compared using the Wilcoxon test. In addition, univariable and multivariable linear regression analyses were used to investigate the preoperative factors that could influence the postoperative hemodynamics, including systolic PAP, Rp, Cp, and the RC time. The preoperative factors investigated included heart rate, the ratio of pulmonary to systemic blood flow (Qp/Qs), Rp, Cp, the RC time, preterm delivery at less than 35 weeks gestational age, low birth weight $(<2.5 \mathrm{~kg})$, and preoperative oxygen supplementation because of airway compromise. Furthermore, the receiver operating characteristic curve was generated by computing sensitivity and specificity at an observed cutoff for adverse postoperative pulmonary hemodynamics. For all statistical analyses, $P$ values $<.05$ were considered significant.

\section{RESULTS}

The demographic data of the patients are shown in Table 1 . The study population consisted of 53 boys and 47 girls. All were delivered at 39 (38-40) weeks gestational age and the median birth weight was $2.8(2.5-3.1) \mathrm{kg}$. Three patients $(3 \%)$ were delivered preterm at less than 35 weeks gestational age and the birth weight of 22 patients $(23 \%)$ was less than $2.5 \mathrm{~kg}$. Three patients who had upper airway obstruction or chronic lung disease had been on oxygen supplement therapy before corrective surgery. No patient was treated or tested with pulmonary vasodilators such as a phosphodiesterase-5 inhibitor or an endothelin receptor antagonist. Morphologic findings included perimembranous ventricular septal defect in 81 patients and subarterial ventricular septal defect in 19 patients. Nine patients had a coexisting atrial septal defect. Patients underwent corrective surgery at a median age of 2.9 (1.8-4.4) months, and most of the patients were infants. The first and second catheterizations were performed at a median age of 2.5 (1.5-3.8) months and 3.5 (2.4-4.9) months, respectively. The first catheterization was performed 9 (5-21) days before surgery, and the second catheterization was performed 13 (12-18) days after surgery.

After surgery, 3 patients received nitric oxide inhalation in the intensive care unit. Home oxygen supplement therapy 
TABLE 1. Patients' demographic data

\begin{tabular}{lc}
\hline \multicolumn{1}{c}{ Characteristic } & Value* \\
\hline Number of patients & 100 \\
Number of boys & 53 \\
Gestational age, wk (range) & $38(38-40)$ \\
Weight at birth, kg (range) & $2.8(2.5-3.1)$ \\
Preoperative oxygen therapy & 3 \\
Preoperative administration of pulmonary vasodilators & 0 \\
Morphology, perimembranous defect & 81 \\
Subarterial defect & 19 \\
Age at preoperative catheter examination, mo (range) & $2.5(1.5-3.8)$ \\
Age at corrective surgery, mo (range) & $2.9(1.8-4.4)$ \\
Age at postoperative catheter examination, mo (range) & $3.5(2.4-4.9)$ \\
Postoperative nitric oxide inhalation & 3 \\
Home oxygen supplement therapy after discharge & 3 \\
Death in hospital & 0 \\
Death in the follow-up period & 1 \\
\hline
\end{tabular}

*Values are the number of patients unless stated otherwise.

was introduced after discharge in 3 patients who had postoperative hypoxia (arterial saturation $<95 \%$ ) or pulmonary arterial systolic pressure greater than $45 \mathrm{~mm} \mathrm{Hg}$. They all discontinued oxygen therapy within 6 months after corrective surgery, because electrocardiography and echocardiography showed improvement of PAH during the follow-up period. There was no in-hospital death but 1 death occurred during the follow-up period as a result of acquired pulmonary venous obstruction.

\section{Changes of Pulmonary Hemodynamics Before and After Surgical Procedure}

Table 2 shows pulmonary hemodynamic parameters before and after surgery. The preoperative Qp/Qs, heart rate, systolic and mean PAP, Rp, Cp, and the RC time were 3.5 (2.7-4.3), 138 (127-147) bpm, 65 (56-70) mm $\mathrm{Hg}, 42$ (36-46) mm Hg, 2.18 (1.64-3.19) Wood unit $/ \mathrm{m}^{2}$, 2.67 (2.01-3.38) $\mathrm{mL} / \mathrm{mm} \mathrm{Hg} / \mathrm{m}^{2}$, and 0.36 (0.31-0.40) seconds, respectively. Figure 1 shows the plots of $\mathrm{Rp}$ and $\mathrm{Cp}$ against the Qp/Qs ratio before surgery. An inverse relationship $(r=-0.57)$ was shown between Qp/Qs and Rp, whereas the relationship between preoperative Qp/Qs and $\mathrm{Cp}$ was directly proportional $(r=0.86)$. These findings show that both $\mathrm{Rp}$ and $\mathrm{Cp}$ are important factors in determining the amount of Qp among these patients.

At the second catheterization, it was confirmed that no residual left-to-right shunt was present after surgery by estimating a postoperative Qp/Qs of 1.0 and performing angiography in all patients. By elimination of the left-toright shunt after corrective surgery, Qp, heart rate, systolic and mean PAP, $\mathrm{Cp}$, and the RC time were significantly decreased to $4.2(3.8-4.7) \mathrm{L} / \mathrm{min} / \mathrm{m}^{2}, 120(111-130) \mathrm{bpm}$ $(P<.0001), 26(23-29) \mathrm{mm} \mathrm{Hg}(P<.0001), 15(13-17)$ $\mathrm{mm} \mathrm{Hg}(P<.0001), 1.96(1.77-2.26) \mathrm{mL} / \mathrm{mm} \mathrm{Hg} / \mathrm{m}^{2}$ $(P<.0001)$, and $.31(.26-0.36)$ seconds $(P<.0001)$, respectively, although $\mathrm{Rp}$ remained unchanged at 2.53 (2.06-3.31) Wood unit $/ \mathrm{m}^{2}$ after surgery $(P=.99)$.

Figure 2, $A$, presents the plots of the relationship between $\mathrm{Rp}$ and $\mathrm{Cp}$ before (closed circles) and after (open circles) surgery. The individual changes before and after surgery are represented by connecting lines (Figure 2, B). The relationship between $\mathrm{Rp}$ and $\mathrm{Cp}$ was inversely related both before and after surgery, which was similar to findings of idiopathic PAH (IPAH) and chronic thromboembolic pulmonary hypertension (CTEPH) in previous reports. ${ }^{4,5}$ When the plots of the logarithm transformation of $\mathrm{Rp}$ against $\mathrm{Cp}$ were drawn, the relationships between $\ln \mathrm{Rp}$ and $\ln \mathrm{Cp}$ were linearly proportional both before and after surgery (Figure 2, $C$ ). The intercept of the approximate line of the relationship between $\ln \mathrm{Rp}$ and $\ln \mathrm{Cp}$, which is a surrogate for the decrease in RC time from 1.60 to 1.10 after surgery. As the RC time decreased with the reduction of Qp, the Rp-Cp coupling curve was shifted downward after corrective surgery. An increase in Qp significantly changed both the RC time and the Rp-Cp coupling.

\section{Preoperative Pulmonary Hemodynamic Parameters that Influence Postoperative Pulmonary \\ Hemodynamics}

Univariable linear regression analysis showed that postoperative pulmonary hemodynamic parameters, including systolic PAP, Rp, and Cp were significantly related to several preoperative systolic PAP, Rp, Cp, and the RC time (Table 3). Particularly, preoperative Qp/Qs (partial regression coefficient, $-1.50 ; 95 \% \mathrm{CI},-2.33$ to -0.68 ;

TABLE 2. Changes in pulmonary hemodynamic parameters before and after surgery

\begin{tabular}{lccc}
\hline & Before surgery & After surgery & $\boldsymbol{P}_{\text {values* }}$ \\
\hline Qp/Qs (range) & $3.5(2.7-4.3)$ & 1.0 & \\
Heart rate, bpm (range) & $138(127-147)$ & $120(111-130)$ & $<.0001$ \\
Systolic PAP, mm Hg (range) & $65(56-70)$ & $26(2-29)$ & $<.0001$ \\
Mean PAP, mm Hg (range) & $42(36-46)$ & $15(13-17)$ & $<.0001$ \\
Rp, Wood unit $/ \mathrm{m}^{2}$ (range) & $2.18(1.64-3.19)$ & $2.53(2.06-3.31)$ & .99 \\
$\mathrm{Cp}, \mathrm{mL} / \mathrm{mm} \mathrm{Hg} / \mathrm{m}^{2}$ (range) & $2.67(2.01-3.38)$ & $1.96(1.77-2.26)$ & $<.0001$ \\
RC time, seconds (range) & $0.36(0.31-0.40)$ & $0.31(0.26-0.36)$ & $<.0001$ \\
\hline
\end{tabular}

$Q p$, Pulmonary blood flow; $Q s$, systemic blood flow; $P A P$, pulmonary arterial pressure; $R p$, pulmonary arterial resistance; $C p$, pulmonary arterial compliance; $R C$, resistorcapacitor. *These variables are compared using the Wilcoxon test. 


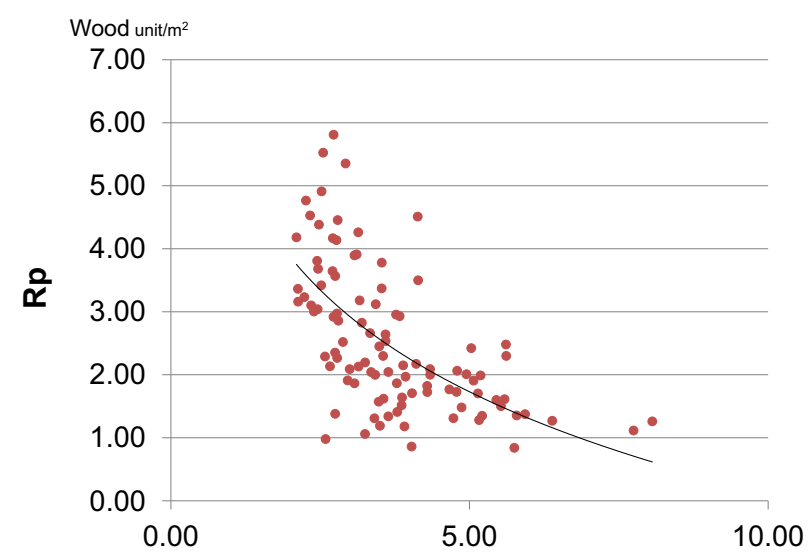

A

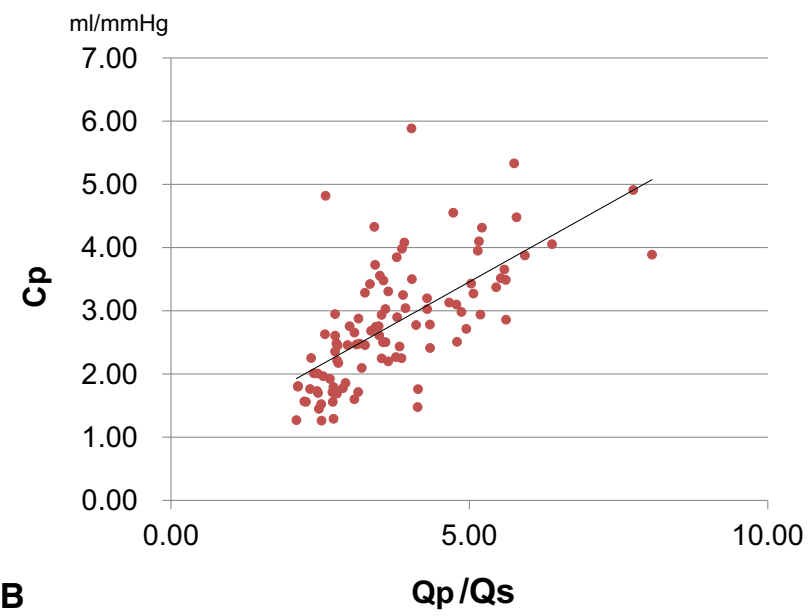

FIGURE 1. The plots of Rp against Qp/Qs show an inverse relationship (A), whereas the plots of $\mathrm{Cp}$ against $\mathrm{Qp} / \mathrm{Qs}$ show a linear proportional relationship (B). $R p$, Pulmonary arterial resistance; $Q p$, pulmonary blood flow; $Q s$, systemic blood flow; $C p$, pulmonary arterial compliance.

$P=.005), \mathrm{Rp}$ (partial regression coefficient, $2.11 ; 95 \% \mathrm{CI}$, $1.28-2.93 ; P<.0001$ ), Cp (partial regression coefficient, $-2.98 ; 95 \% \mathrm{CI},-3.90$ to $-2.05 ; P<.0001$ ), and preterm delivery (partial regression coefficient, $11.00 ; 95 \% \mathrm{CI}$, $5.23-16.76 ; P=.0003$ ) were significantly related to postoperative systolic PAP. However, multivariable linear regression analysis showed that preoperative $\mathrm{Cp}$ (partial regression coefficient, $-2.69 ; 95 \% \mathrm{CI},-5.09$ to -0.29 ; $P=.02$ ) and preterm delivery (partial regression coefficient, $6.67 ; 95 \% \mathrm{CI}, 1.07-12.27 ; P=.02$ ) were significantly related to postoperative systolic PAP. The partial regression coefficient for heart rate was negligible. Therefore, lower preoperative $\mathrm{Cp}$ and preterm delivery at less than 35 weeks were independent predictors for higher postoperative systolic PAP.

The receiver operating characteristic curve was generated using preoperative $\mathrm{Cp}$ values as the predictor of postoperative systolic PAP. For prediction of postoperative
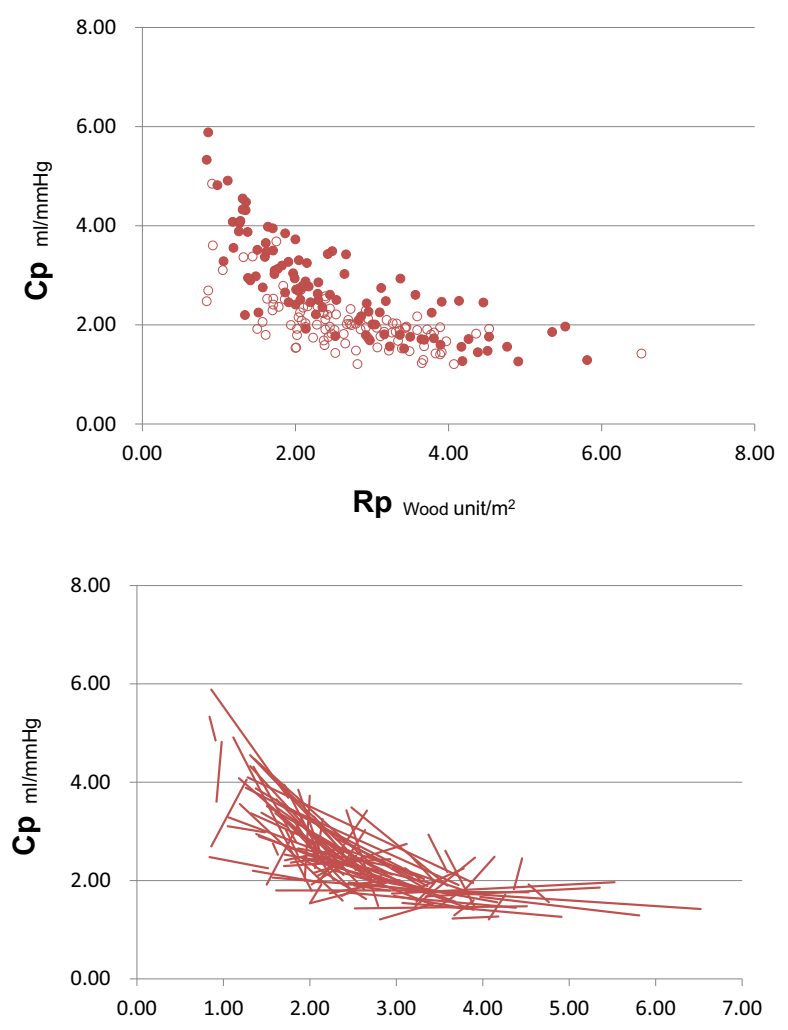

B

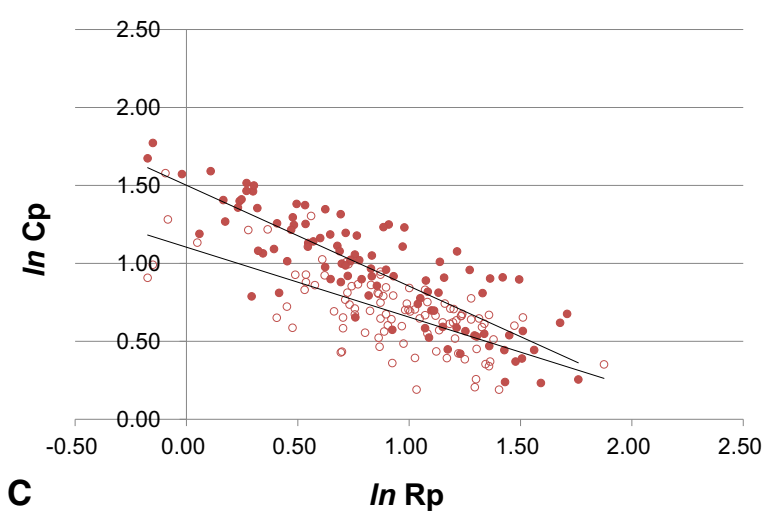

FIGURE 2. The relationship between pulmonary arterial resistance $(R p)$ and compliance $(C p)$ before (closed circles) and after (open circles) surgery (A); individual changes are shown as connecting lines (B). The plots of the logarithms of $\mathrm{Rp}(\ln R p)$ and $\mathrm{Cp}(\ln C p)$ show a linear relationship (C).

systolic PAP greater than $30 \mathrm{~mm} \mathrm{Hg}$, a Cp cutoff value of less than $2.50 \mathrm{~mL} / \mathrm{mm} \mathrm{Hg} / \mathrm{m}^{2}$ yielded a sensitivity and a specificity of $77 \%$ and $67 \%$, respectively. The area under the curve was 0.74 . On the other hand, a Cp cutoff value of less than $2.21 \mathrm{~mL} / \mathrm{mm} \mathrm{Hg} / \mathrm{m}^{2}$ yielded a sensitivity and a specificity of $78 \%$ and $100 \%$, respectively, to predict postoperative systolic PAP greater than $40 \mathrm{~mm} \mathrm{Hg}$ (area under the curve was 0.83 ). Three patients who showed postoperative systolic PAP greater than $40 \mathrm{~mm} \mathrm{Hg}$ had preoperative $\mathrm{Cp}$ less than $2.00 \mathrm{~mL} / \mathrm{mm} \mathrm{Hg} / \mathrm{m}^{2}$. Lower 
TABLE 3. Univariable and multivariable analysis between preoperative and postoperative hemodynamic parameters

\begin{tabular}{|c|c|c|c|c|c|c|c|}
\hline \multirow{2}{*}{$\begin{array}{c}\text { Dependent variable } \\
\begin{array}{c}\text { Postoperative } \\
\text { parameter }\end{array}\end{array}$} & \multirow{2}{*}{$\begin{array}{c}\text { Independent variable } \\
\text { Preoperative parameter }\end{array}$} & \multicolumn{3}{|c|}{ Univariable analysis } & \multicolumn{3}{|c|}{ Multivariable analysis } \\
\hline & & Coefficient & $95 \% \mathbf{C I}$ & $P$ value & Coefficient & $\mathbf{9 5} \% \mathbf{C I}$ & $P$ value \\
\hline \multirow[t]{9}{*}{ Systolic PAP } & Qp/Qs & -1.50 & -2.33 to -0.68 & $.005^{*}$ & & & \\
\hline & Heart rate & -0.11 & -0.19 to -0.04 & $.002 *$ & -.11 & -0.17 to -0.04 & $.001 *$ \\
\hline & Systolic PAP & 0.16 & 0.08 to 0.24 & $.0001 *$ & & & \\
\hline & $\mathrm{Rp}$ & 2.11 & 1.28 to 2.93 & $<.0001 *$ & & & \\
\hline & $\mathrm{Cp}$ & -2.98 & -3.90 to -2.05 & $<.0001 *$ & -3.35 & -5.33 to -1.38 & $.001 *$ \\
\hline & $\mathrm{RC}$ time & 0.56 & -0.09 to 1.22 & ns & & & \\
\hline & Preterm delivery & 11.00 & 5.23 to 16.76 & $.0003 *$ & 6.28 & 0.71 to 11.85 & $.02 *$ \\
\hline & Low birth weight & 1.03 & -1.50 to 3.57 & ns & & & \\
\hline & Preoperative oxygen supplement & 9.86 & 2.61 to 17.12 & $.008^{*}$ & & & \\
\hline \multirow[t]{9}{*}{$\mathrm{Rp}$} & $\mathrm{Qp} / \mathrm{Qs}$ & -0.16 & -0.30 to -0.01 & $.03^{*}$ & & & \\
\hline & Heart rate & -0.01 & -0.02 to 0.00 & ns & & & \\
\hline & Systolic PAP & 0.02 & 0.00 to 0.03 & $.007^{*}$ & & & \\
\hline & $\mathrm{Rp}$ & 0.34 & 0.20 to 0.49 & $<.0001 *$ & & & \\
\hline & $\mathrm{Cp}$ & -0.32 & -0.49 to -0.14 & $.006^{*}$ & & & \\
\hline & $\mathrm{RC}$ time & 0.19 & 0.07 to 0.29 & $.001 *$ & & & \\
\hline & Preterm delivery & 2.01 & 1.02 to 2.99 & $.0001 *$ & 1.91 & 0.82 to 3.00 & $.0008 *$ \\
\hline & Low birth weight & 0.77 & -0.36 to 0.52 & ns & & & \\
\hline & Preoperative oxygen supplement & 0.48 & -0.81 to 1.78 & $\mathrm{~ns}$ & & & \\
\hline \multirow[t]{9}{*}{$\mathrm{Cp}$} & $\mathrm{Qp} / \mathrm{Qs}$ & 0.14 & 0.05 to 0.23 & $.001 *$ & & & \\
\hline & Heart rate & 0.01 & 0.00 to 0.01 & ns & & & \\
\hline & Systolic PAP & -0.02 & -0.03 to -0.01 & $<.0001 *$ & & & \\
\hline & $\mathrm{Rp}$ & -0.24 & -0.33 to -0.16 & $<.0001 *$ & & & \\
\hline & $\mathrm{Cp}$ & 0.35 & 0.25 to 0.44 & $<.0001 *$ & 0.55 & 0.29 to 0.80 & $.00001 *$ \\
\hline & $\mathrm{RC}$ time & -0.08 & -0.15 to 0.01 & $.029 *$ & & & \\
\hline & Preterm delivery & -0.77 & -1.40 to -0.14 & $.017^{*}$ & & & \\
\hline & Low birth weight & 0.00 & -0.23 to 0.30 & $\mathrm{~ns}$ & & & \\
\hline & Preoperative oxygen supplement & -0.55 & -1.33 to 0.24 & $\mathrm{~ns}$ & & & \\
\hline \multirow[t]{9}{*}{ RC time } & Qp/Qs & 0.02 & -0.20 to 0.24 & ns & & & \\
\hline & Heart rate & -0.01 & -0.03 to 0.01 & ns & & & \\
\hline & Systolic PAP & 0.01 & -0.01 to 0.03 & ns & & & \\
\hline & $\mathrm{Rp}$ & 0.16 & -0.07 to 0.39 & $\mathrm{~ns}$ & & & \\
\hline & $\mathrm{Cp}$ & 0.07 & -0.21 to 0.34 & ns & & & \\
\hline & $\mathrm{RC}$ time & 0.25 & 0.09 to 0.41 & $.0024 *$ & & & \\
\hline & Preterm delivery & 1.02 & -0.51 to 2.56 & ns & & & \\
\hline & Low birth weight & -0.10 & -0.74 to 0.53 & ns & & & \\
\hline & Preoperative oxygen supplement & -0.06 & -2.44 to 1.33 & $\mathrm{~ns}$ & & & \\
\hline
\end{tabular}

$C I$, Confidence interval; $P A P$, pulmonary arterial pressure; $Q p$, pulmonary blood flow; $Q s$, systemic blood flow; $R p$, pulmonary arterial resistance; $C p$, pulmonary arterial compliance; $R C$, resistor-capacitor; $n s$, not significant. *Statistical significance.

preoperative $\mathrm{Cp}$ can predict higher postoperative PAP independently, which should be useful in the management of patients with left-to-right shunt congenital heart disease.

\section{DISCUSSION}

We show that an increase in Qp leads to an increase in RC time and shifts the Rp-Cp inverse relation curve upward. When discussing pulmonary circulation, including Rp and $\mathrm{Cp}$, among patients with $\mathrm{PAH}$ related to left-to-right shunt congenital heart disease, it is important to consider 2 factors that influence the change in the Rp-Cp coupling in the pulmonary circuit. One is the inverse relationship between $\mathrm{Rp}$ and $\mathrm{Cp}$ depending on the condition of the pulmonary vasculature, which represents the change in the Rp-Cp coupling curve. The other is the change in the RC time depending on the amount of Qp, which represents the shift of the Rp-Cp coupling curve (Figure 3 ). Therefore, the changes in $\mathrm{Rp}$ and $\mathrm{Cp}$ before and after surgery are variable in individuals, as shown in Figure 2, B. In addition, we reveal that lower preoperative $\mathrm{Cp}$ and preterm delivery can be independent predictors of higher postoperative systolic PAP. To our knowledge, this is the first study showing the change in $\mathrm{Rp}-\mathrm{Cp}$ coupling in $\mathrm{PAH}$ and congenital heart disease before and after corrective surgery.

The pulmonary circuit reacts as a series of resistercapacitor circuits and consists of resistant vessels and capacitance vessels. Hence, the pulmonary circuit is characterized as a low-pressure circuit with normal arterial 


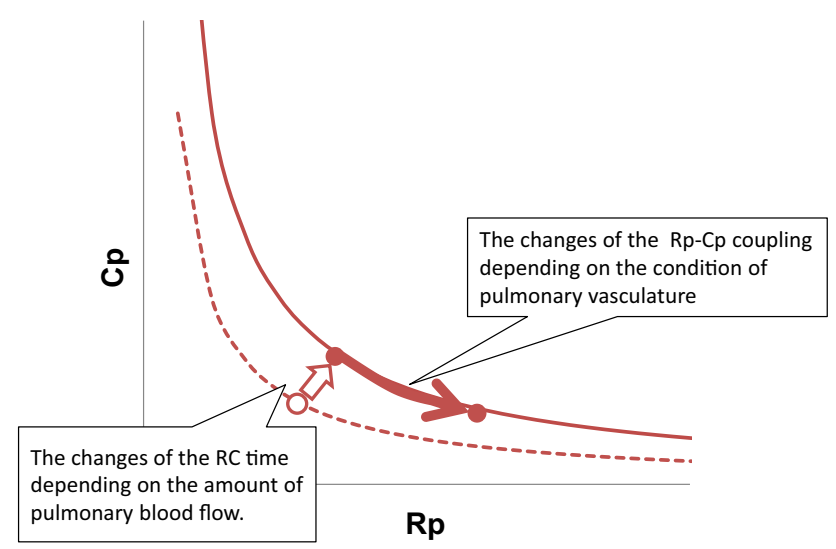

FIGURE 3. The relationship between pulmonary arterial resistance $(R p)$ and compliance $(C p)$ is influenced by the amount of pulmonary blood flow and the condition of the pulmonary vasculature. $R C$, Resistorcapacitor.

pressure as low as about $15 \mathrm{~mm} \mathrm{Hg}$, despite receiving high blood volume proportional to the entire cardiac output. The $\mathrm{RC}$ time in the pulmonary circulation was previously shown to be fixed during therapy in patients with PAH. However, it has been reported that the RC times vary depending on the nature or cause of PAH. When RC times are compared between IPAH and CTEPH, the RC time is higher in patients with IPAH than in those with CTEPH. ${ }^{4,5}$ However, the reason why $\mathrm{RC}$ time varies between the diseases has been ambiguous. In the present study, we show that the increase in Qp leads to an increase in the RC time. Because the reduction of Qp after corrective surgery leads to a decrease in $\mathrm{Cp}$ and no change in $\mathrm{Rp}$, the change in the $\mathrm{RC}$ time predominantly depends on the change in $\mathrm{Cp}$.

The following reasons may explain why increased Qp affects $\mathrm{Cp}$ among patients with PAH related to left-to-right shunt congenital heart disease. First, the increase in Qp leads to pulmonary capillary recruitment. ${ }^{6}$ Pulmonary capillaries are partially or completely collapsed in the top part of the lungs during normal Qp, but these collapsed vessels are recanalized as Qp and pulmonary pressure increase. This process is attributed to the fact that pulmonary capillaries are exceedingly thin and compliant. Therefore, an amplified pulmonary arterial pulse caused by excessive Qp augments systolic PAP, which results in an increase of total $\mathrm{Cp}$ in patients with left-to-right shunt. Second, it is possible that structural changes in proximal pulmonary arteries also affect $\mathrm{Cp}$. The previous study revealed that $\mathrm{Cp}$ was increased after percutaneous endarterectomy in patients with proximal CTEPH, which was explained by the improvements in the elasticity of proximal pulmonary arteries after the removal of obstructions. The elasticity of the proximal pulmonary arteries contributes $15 \%$ to $20 \%$ of total Cp. ${ }^{7}$ The increase in Qp leads to dilation of the proximal pulmonary arteries, which are more elastic because of thin arterial walls in infants. The elasticity of the pulmonary artery appears to be as important as the state of the arterioles and capillaries in determining the energy required for pulsatile Qp.

We show that $\mathrm{Rp}$ remains unchanged before and after corrective surgery, although previous reports describe decreased Rp after corrective surgery in patients with leftto-right shunt congenital heart disease. Because our study cohort population consists of infants at the median age of 2.9 months, pulmonary vascular obstructive disease caused by excessive Qp has not yet developed in these patients because the median value of $\mathrm{Rp}$ is 2.18 (1.64-3.19) Wood unit $/ \mathrm{m}^{2}$. In the present study, furthermore, postoperative pulmonary hemodynamics were measured as early as 13 (12-18) days after the surgery, whereas postoperative $\mathrm{Rp}$ tended to still be higher because of the damage to the pulmonary vasculature after use of cardiopulmonary bypass.

Multivariable linear regression analysis shows that a lower preoperative $\mathrm{Cp}$ can be an independent predictor of higher postoperative systolic PAP. A recent study showed that both $\mathrm{Rp}$ and $\mathrm{Cp}$ are strong independent predictors for outcomes in pediatric IPAH. ${ }^{8}$ Therefore, both Rp and Cp should be considered when assessing surgical outcomes or morbidity in patients with PAH related to left-to-right congenital heart disease, although surgical outcomes in patients with ventricular septal defect are extremely favorable. We have encountered a few patients who have unexpected pulmonary hypertension and need oxygen therapy even after corrective surgery. The present study suggests that aggressive assessments of pulmonary hemodynamics after surgery, such as cardiac catheterization, are warranted among patients with lower preoperative $\mathrm{Cp}$.

We show that preterm delivery is another predictor for higher postoperative systolic PAP. It is acknowledged that preterm infants with chronic lung disease are complicated with PAH because of maldevelopment of the alveoli and abnormal organization of vascular structures. The incidence of congenital heart disease is 12.5 per 1000 births in preterm infants, and $8 \%$ to $25 \%$ of preterm infants have chronic lung disease. Therefore, we estimate that at least $1.2 \%$ to $4 \%$ of infants with congenital heart disease have concomitant chronic lung disease. ${ }^{9}$ However, in the clinical setting, the clinical findings do not distinguish whether PAH is related to left-to-right shunt congenital heart disease or chronic lung disease among such premature infants.

\section{Study Limitations}

In practice, calculation of compliance is difficult because blood is leaving the arterial system through the periphery while cardiac ejection occurs. There are 2 methods to calculate $\mathrm{Cp}$. One is the pulse pressure method, which uses the 2-element Windkessel model with flow waveform and resistance as inputs to estimate the compliance value that best predicts systolic and diastolic pressures. The other method is derived from the exponential decay of the diastolic PAP 
wave method in vivo. Previous studies ${ }^{6,7}$ have explained the observed differences in the RC time constant as being a result of different methods of calculating compliance. In the present study, we have chosen the pulse pressure method to calculate total $\mathrm{Cp}$, because our study is retrospective, using the parameters available in right heart catheterization.

\section{CONCLUSIONS}

The relationship between Rp and Cp was inverse among infants with $\mathrm{PAH}$ associated with left-to-right shunt congenital heart disease. Rp-Cp couplings varied depending on Qp and the condition of the pulmonary vasculature. We showed that preoperative $\mathrm{Cp}$ can be an independent predictor of adverse postoperative hemodynamics, and lower preoperative $\mathrm{Cp}$ is related to higher postoperative systolic PAP. In addition, a preoperative $\mathrm{Cp}$ of less than $2.21 \mathrm{~mL} / \mathrm{mm} \mathrm{Hg} / \mathrm{m}^{2}$ can predict postoperative systolic PAP greater than $40 \mathrm{~mm} \mathrm{Hg}$. Many pediatric cardiologists and cardiovascular surgeons are unfamiliar with the concept of $\mathrm{Rp}-\mathrm{Cp}$ coupling in the management of congenital heart disease. Our findings should facilitate the comprehensive management of patients with PAH associated with congenital heart disease.

\section{Conflict of Interest Statement}

Authors have nothing to disclose with regard to commercial support.
We would like to thank Editage (www.editage.jp) for English language editing.

\section{References}

1. Sagawa K, Lie RK, Schaefer J. Translation of Otto Frank's paper "Die Grundform des Arteriellen Pulses" Zeitschrift fur Biologie. 37: 483-526 (1899). J Mol Cell Cardiol. 1990;22:253-4

2. Lankhaar JW, Westerhof N, Faes TJ, Marques KM, Marcus JT, Postmus PE, et al Quantification of right ventricular afterload in patients with and without pulmonary hypertension. Am J Physiol Heart Circ Physiol. 2006;291:1731-7.

3. Saouti N, Westerhof N, Postmus PE, Vonk-Noordegraaf A. The arterial load in pulmonary hypertension. Eur Respir Rev. 2010;19:197-203.

4. Lankhaar JW, Westerhof N, Faes TJ, Gan CT, Marques KM, Boonstra A, et al. Pulmonary vascular resistance and compliance stay inversely related during treatment of pulmonary hypertension. Eur Heart J. 2008;29:1688-95.

5. MacKenzie Ross RV, Toshner MR, Soon E, Naeije R, Pepke-Zaba J. Decreased time constant of the pulmonary circulation in chronic thromboembolic pulmonary hypertension. Am J Physiol Heart Circ Physiol. 2013;305:259-64.

6. Presson RG Jr, Baumgartner WA Jr, Peterson AJ, Glenny RW, Wagner WW Jr. Pulmonary capillaries are recruited during pulsatile flow. J Appl Physiol. 2002;92: 1183-90.

7. Saouti N, Westerhof N, Helderman F, Marcus JT, Stergiopulos N, Westerhof BE et al. RC time constant of single lung equals that of both lungs together: a study in chronic thromboembolic pulmonary hypertension. Am J Physiol Heart Circ Physiol. 2009;297:2154-60.

8. Douwes JM, Roofthooft MT, Bartelds B, Talsma MD, Hillege HL, Berger RM Pulsatile haemodynamic parameters are predictors of survival in paediatric pulmonary arterial hypertension. Int J Cardiol. 2013;168:1370-7.

9. Muneuchi J, Kuraoka A, Watanabe M, Ochiai Y, Joo K. Pulmonary arterial hypertension associated with bronchopulmonary dysplasia and congenital heart disease in preterm infants. A case report of a preterm infant with recurrent pulmonary hypertension after corrective cardiac surgery and review of the literature. Int Heart J. 2015;56(suppl):S22-5.

Key Words: resistance, compliance, RC time constant, leftto-right shunt, pulmonary arterial hypertension 